\title{
Complicaciones en cirugía ginecológica laparoscópica en un hospital público de tercer nivel
}

\section{Complications in laparoscopic gynecological surgery in a tertiary-level public hospital}

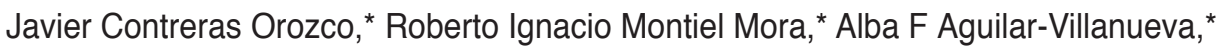 \\ Fabiola Brito Sandoval, ${ }^{*}$ Gamaliel Rodríguez Reyes, ${ }^{\ddagger}$ Javier Benítez Beltrán ${ }^{\ddagger}$ \\ * Cirugía Endoscópica Ginecológica. Facultad Mexicana de Medicina, Universidad La Salle. México. \\ ₹Curso de Alta Especialidad en Cirugía Endoscópica Ginecológica.
}

\section{RESUMEN}

Introducción: El abordaje laparoscópico ha revolucionado el campo de la cirugía ginecológica con beneficios que incluyen disminución del dolor posoperatorio, estancia intrahospitalaria más corta, reincorporación temprana de actividades normales y menor número de complicaciones posoperatorias en comparación con la técnica abierta. Objetivo: Comparar la relación entre el porcentaje de complicaciones en un hospital público de tercer nivel con la literatura médico-científica. Material y métodos: Diseño observacional transversal retrospectivo. Se revisaron y analizaron los expedientes clínicos de pacientes con cirugías laparoscópicas ginecológicas, se obtuvo el porcentaje de complicaciones totales. Los procedimientos se llevaron a cabo en el periodo de marzo de 2018 a septiembre de 2020 en el Hospital Regional «Licenciado Adolfo López Mateos» del ISSSTE. Se realizaron en total 117 cirugías. Resultados: Se observaron 15 complicaciones entre los 117 procedimientos ginecológicos laparoscópicos, de los cuales sólo 13 se relacionan con complicaciones menores y dos (lesión vascular e intestinal) a complicaciones mayores. Sin reporte de secuela posquirúrgica o mortalidad. Conclusiones: En nuestra experiencia institucional, las únicas complicaciones a las que nos enfrentamos fueron lesión de vaso epigástrico, lesión intestinal, hemorragia mayor de la esperada, dolor posquirúrgico relacionado y no relacionado con sitios de punción y hernia posincisional; ninguna de las complicaciones presentó una incidencia mayor de $2.5 \%$, lo que nos sitúa dentro del promedio e, incluso, por debajo en ciertas complicaciones, comparado con lo reportado en la literatura médica.

Palabras clave: Complicaciones, laparoscopía ginecológica.

\section{ABSTRACT}

Introduction: Laparoscopic approach has revolutionized the field of gynecological surgery with benefits that include decreased postoperative pain, shorter days of hospital stay, early reincorporation of normal activities and fewer postoperative complications compared to the open technique. Objective: Compare the relationship between the percentage of complications in a third level public hospital with the medical-scientific literature. Material and methods: Retrospective cross-sectional observational design, the clinical records of patients with gynecological laparoscopic surgeries were reviewed and analyzed, obtaining the percentage of total complications. The procedures were carried out in the period from March 2018 to September 2020 at the "Adolfo López Mateos" ISSSTE Regional Hospital, undergoing a total of 117 surgeries. Results: Fifteen complications were observed from 117 laparoscopic gynecological procedures, of which only 13 are related to minor complications and two (vascular and intestinal injury) to major complications. No report of postsurgical sequel or mortality. Conclusions: In our institutional experience, the only complications we faced were epigastric vessel injury, intestinal injury, major bleeding, expected post-surgical pain related and not related to puncture sites and post incisional hernia, none of the complications presented an incidence greater than $2.5 \%$ being within the average and even below in certain complications according to medical literature.

Keywords: Complications, gynecological laparoscopy. 


\section{INTRODUCCIÓN}

El abordaje laparoscópico ha revolucionado el campo de la cirugía ginecológica. En comparación con la cirugía abierta, la vía laparoscópica promete beneficios que incluyen disminución del dolor posoperatorio, estancia intrahospitalaria más corta, reincorporación temprana a actividades normales y menor número de complicaciones posoperatorias.

Las complicaciones asociadas con la laparoscopía ginecológica no son frecuentes, tienen una incidencia de tres a seis por cada 1,000 casos y una tasa de mortalidad de 3.3 por cada $100,000 .^{1}$

La cirugía laparoscópica por sí sola se le ha relacionado con complicaciones específicas en la literatura mundial. Estas complicaciones incluyen problemas anestésicos y tromboembólicos, hemorragia e infección. Esto sin omitir las complicaciones inherentes a cada proceso quirúrgico. Se identifican prominentes las complicaciones asociadas con la técnica de entrada a cavidad abdominal, neumoperitoneo y relacionadas con la restricción del campo visual en algunos casos. A la vez, estas últimas pueden clasificarse en mayores o menores. Las mayores incluyen lesiones viscerales o vasculares, mientras que las menores, por lo general, tienen un impacto leve en el curso posquirúrgico del paciente. ${ }^{2}$

Las complicaciones derivadas de las técnicas de ingreso y neumoperitoneo son las más frecuentes. Las lesiones vasculares tienen una incidencia de 0.4 en 1,000 procedimientos con una tasa de mortalidad de 9 a $17 \%{ }^{3,4}$ Las intestinales son más habituales que las lesiones vasculares de mayor calibre, con una incidencia de 0.7-1.1/1,000 procedimientos; ${ }^{4}$ se pueden clasificar como lesiones tipo 1 cuando el intestino está en su posición anatómica normal y lesiones tipo II cuando el intestino se encuentra adherido a la pared abdominal. ${ }^{5}$ También se incluyen daños a estructuras retroperitoneales, insuflación extraperitoneal y herniación a través de puertos.

\section{MATERIAL Y MÉTODOS}

Se realizó un estudio observacional transversal retrospectivo, se revisó y analizó el expediente clínico de 117 pacientes del Hospital Regional «Licenciado Adolfo López Mateos» del ISSSTE, a quienes se les realizaron procedimientos laparoscópicos ginecológicos en el periodo de marzo de 2018 a septiembre de 2020. Se tomaron como criterios de inclusión que los pacientes ameritaran, por clínica, un procedimiento quirúrgico ginecológico laparoscópico, que contaran con valoración por parte del Servicio de Medicina Interna para entrar de manera segura a quirófano y que tuvieran expediente clínico activo en el hospital. Se excluyó a quienes no contaran con expediente clínico completo o que no se resolvieran quirúrgicamente por vía laparoscópica. De acuerdo con lo observado en los procedimientos, se tabularon las observaciones hacia las tablas de resultados finales.

\section{RESULTADOS}

Fueron incluidas 117 pacientes, se les realizaron los siguientes procedimientos quirúrgicos ginecológicos: histerectomía total laparoscópica, miomectomía laparoscópica, salpingectomía bilateral laparoscópica, cistectomía laparoscópica, neurectomía presacra laparoscópica, ablación de ligamentos uterosacros laparoscópica y laparoscopía diagnóstica. Se revisó si existían complicaciones en dichos procedimientos, incluidas lesiones a estructuras adyacentes, hemorragia mayor de la esperada $(<500 \mathrm{~mL})$ cuantificada por el Servicio de Anestesiología, conversión a laparotomía, ingreso a la Unidad de Cuidados Intensivos del Adulto (UCIA), dolor posquirúrgico en sitios de punción moderado/severo (utilizando escala visual EVA, considerando una puntuación mayor de 6), dolor posquirúrgico no relacionado con sitios de punción (dolor en hombro derecho), retraso de alta hospitalaria (mayor de 72 horas posquirúrgicas), insuflación extraperitoneal, hernia posincisional y, en el caso de histerectomías, la presencia de dehiscencia de cúpula vaginal. Esto con el objetivo de comparar la relación entre el porcentaje de complicaciones en un hospital público de tercer nivel con la literatura médico-científica.

Las cirugías laparoscópicas realizadas se muestran en la tabla 1, incluyeron 65 histerectomías totales laparoscópicas, 26 cistectomías laparoscópicas y ocho laparoscopías diagnósticas. La tabla 2 presenta las complicaciones registradas en las cirugías laparoscópicas realizadas.

\section{DISCUSIÓN}

La lesión asociada con la entrada laparoscópica ha sido motivo de estudio durante décadas, con innovación técnica e intento de consenso, ya que una cantidad significativa

\begin{tabular}{|lcc|}
\multicolumn{3}{|c|}{ Tabla 1: Cirugías laparoscópicas. } \\
\hline Cirugía & $\mathrm{n}$ & $\%$ \\
\hline Histerectomía total laparoscópica & 65 & 55.5 \\
Cistectomía laparoscópica & 26 & 22.2 \\
Laparoscopía diagnóstica & 8 & 6.8 \\
Miomectomía laparoscópica & 7 & 5.9 \\
Salpingectomía bilateral laparoscópica & 6 & 5.1 \\
Ablación de ligamentos uterosacros & 4 & 3.4 \\
laparoscópica & & \\
Neurectomía presacra laparoscópica & 1 & 0.8 \\
\hline
\end{tabular}


Tabla 2: Complicaciones de cirugías laparoscópicas.

\begin{tabular}{|c|c|c|}
\hline Complicación & $n$ & $\%$ \\
\hline $\begin{array}{l}\text { Lesiones vasculares (incluyendo de } \\
\text { pared abdominal) }\end{array}$ & 1 & 0.8 \\
\hline Lesiones de vías urinarias & 0 & 0.0 \\
\hline Lesión intestinal & 1 & 0.8 \\
\hline Hemorragia (mayor de $500 \mathrm{~mL}$ ) & 2 & 1.7 \\
\hline Insuflación extraperitoneal & 0 & 0.0 \\
\hline Ingreso a la UCIA & 0 & 0.0 \\
\hline $\begin{array}{l}\text { Dolor posquirúrgico en sitios de pun- } \\
\text { ción moderado/severo }\end{array}$ & 2 & 1.7 \\
\hline $\begin{array}{l}\text { Dolor posquirúrgico no relacionado } \\
\text { con sitios de punción }\end{array}$ & 3 & 2.5 \\
\hline Retraso de alta hospitalaria & 2 & 1.7 \\
\hline Hernia posincisional & 2 & 1.7 \\
\hline Infección en herida quirúrgica & 2 & 1.7 \\
\hline Dehiscencia de cúpula vaginal & 0 & 0.0 \\
\hline
\end{tabular}

de todas las complicaciones ocurren en el momento de la entrada a la pared abdominal, con una tasa general de lesiones relacionadas con entrada de 3.3/1,000. ${ }^{6}$

Las lesiones vasculares son las complicaciones más graves debido a su inmediatez y a la posible mortalidad. La mayoría de las lesiones vasculares ocurren durante la entrada al peritoneo con la inserción del trocar o la aguja de Veress; sin embargo, también se informa que ocurren con técnicas de entrada directa y abierta. La aorta y los vasos iliacos comunes derechos están en alto riesgo de lesión, debido a su proximidad al ombligo en la entrada inicial. Las lesiones de los vasos epigástricos inferiores y sus ramas son más comunes con la colocación de un puerto secundario y las lesiones de estos vasos también han provocado consecuencias mortales. En nuestro estudio encontramos una incidencia de lesiones vasculares de $0.8 \%$, únicamente presentamos una lesión a vaso epigástrico, siendo ésta la más lesionada al momento de la entrada de trocares laterales, con una frecuencia de $0.2-2 \%$ reportada en la literatura. ${ }^{4}$

Las lesiones gastrointestinales pueden ocurrir en cualquier víscera, incluidos el esófago y el estómago, pero con mayor frecuencia se producen en el intestino delgado con una tasa de $55.8 \%$, seguido del intestino grueso con $38.6 \%{ }^{7}$ Las lesiones viscerales se producen con cualquier técnica de entrada y con trocares primarios y secundarios. A menudo se considera que la lesión de un trocar primario puede ser inevitable con una lesión intestinal de tipo II; no obstante, la opinión es tal que la lesión de un puerto secundario debería ser evitable.

Múltiples estudios informan que la inserción de trocar primario o aguja de Veress da como resultado aproximada- mente $50 \%$ de todas las lesiones intestinales laparoscópicas. Una revisión de 2004 con 329,935 pacientes detectó una incidencia global de lesión intestinal en laparoscopía de $0.13 \%$, con $0.22 \%$ en lesiones penetrantes. ${ }^{7}$ Una serie retrospectiva reciente de cinco años reveló una incidencia de $0.11 \%$ en lesiones intestinales. ${ }^{8}$ Nuestra incidencia de lesión intestinal fue de $0.8 \%$, en donde la lesión se presentó en intestino delgado en una histerectomía total laparoscópica provocada por una pinza grasper, la cual se diagnosticó y reparó durante el mismo procedimiento quirúrgico por laparoscopía, la reparación se realizó con vicryl 2-0 en dos planos. La paciente presentó como comorbilidad retraso al momento del alta.

Las lesiones intestinales de tipo I, daño con vísceras en posición anatómica, ocurren rara vez con técnicas de entrada abierta o cerrada cuando se sigue el procedimiento correcto. Sin embargo, cuando el intestino está anormalmente posicionado en el peritoneo (lesiones de tipo II), por lo regular como resultado de adherencias, es difícil evitar la lesión con cualquier técnica de entrada como resultado de la posición inesperada del intestino al entrar en la cavidad. En este momento, el reconocimiento de la lesión y la reparación adecuada es clave para evitar una morbilidad significativa, e incluso la mortalidad.

La incidencia de hernia de intestino a través de un sitio de punción (hernia de Richter) es poco común y está relacionada con el tamaño del puerto. Se reporta que ocurre en 21 de 100,000 procedimientos laparoscópicos y en una tasa mucho menor que la asociada con la incisión de laparotomía. ${ }^{9}$ Las hernias laparoscópicas en el sitio del puerto son más comunes en forma lateral que central, y el riesgo está directamente relacionado con el tamaño del puerto, dicho riesgo aumenta con un tamaño de puerto más grande. Se ha encontrado que la hernia rara vez ocurre en puertos de 5 y de $7 \mathrm{~mm}$, y es más común en puertos de más de $10 \mathrm{~mm}$, con un aumento de $3.1 \%$ de riesgo en puertos de $12 \mathrm{~mm}$ de tamaño. ${ }^{10}$ Una hernia de Richter incisional puede conducir a la muerte si no se reconoce en el momento del procedimiento o posoperatoriamente. Entre nuestras complicaciones observamos una incidencia de $1.7 \%$, ambas pacientes fueron sometidas a miomectomía laparoscópica. La hernia se presentó en el sitio del puerto paraumbilical derecho que se amplió a $3 \mathrm{~cm}$ para la morcelación y extracción manual de la pieza quirúrgica.

Se espera dolor por la colocación del trocar, pero puede minimizarse, utilizando la menor cantidad de puertos necesarios para realizar el procedimiento de manera segura, minimizando el número de puertos grandes $(\geq 10 \mathrm{~mm})$ e inyectando anestesia local en los sitios del puerto antes o después del procedimiento. A todas nuestras pacientes se les infiltraron los puertos al final del procedimiento con 
ropivacaína $7.5 \mathrm{mg} / \mathrm{mL}$, y se presentó una incidencia de dolor posquirúrgico en sitios de punción de 1.7\%.

Una revisión sistemática de las técnicas de histerectomía incluye un metaanálisis de 34 estudios con 4,495 mujeres, dominado por el estudio eVALuate (Vaginal-Abdominal-Laparoscópico) a gran escala que reporta asociación estadísticamente significativa de más lesiones del tracto urinario en el grupo de histerectomía laparoscópica $(\mathrm{HL})$, comparado con el de histerectomía abierta (OR 3.69, IC del 95\% 1.11-12.24). ${ }^{11}$ Este aumento podría ser el resultado de la anatomía, la patología tisular, la técnica como sutura versus energía o la experiencia del cirujano en el procedimiento. Sin embargo, existe un sesgo continuo inherente en estos datos, ya que los análisis siempre incluirán datos más antiguos y esto tiene el efecto de reducir la probabilidad de complicaciones oblicuas para estudios adicionales más pequeños. En nuestra revisión no se encontraron lesiones urinarias.

La hemorragia puede ocurrir de manera intraoperatoria o posoperatoria y puede ser consecuencia de una lesión del peritoneo, estructuras urológicas o de los principales vasos sanguíneos. Una revisión Cochrane de las técnicas de histerectomía evaluó la hemorragia resultante y en los datos se encontró que se compararon histerectomía laparoscópica e histerectomía abdominal (HA), las tasas de hemorragia sustancial fueron menores con la técnica laparoscópica. ${ }^{12}$

\section{CONCLUSIONES}

A medida que avance la tecnología y mejoren los entrenamientos y destrezas de los ginecólogos laparoscopistas, disminuirá el número de complicaciones presentadas. En nuestra experiencia institucional, las únicas complicaciones a las que nos enfrentamos fueron lesión de vaso epigástrico, lesión intestinal, hemorragia mayor de la esperada, dolor posquirúrgico relacionado y no relacionado con sitios de punción y hernia posincisional; sin embargo, ninguna de las complicaciones presentó una incidencia mayor de $2.5 \%$, lo que nos sitúa dentro del promedio e incluso por debajo en ciertas complicaciones en comparación con lo informado en la literatura médica.

\section{REFERENCIAS}

1. Sepúlveda-Agudelo J. Complicaciones laparoscópicas asociadas a la técnica de entrada. Rev Colomb Obstet Ginecol. 2011; 62: 88-93.

2. Fuentes MN, Rodríguez-Oliver A, Naveiro Rilo JC, Paredes AG, Aguilar Romero MT, Parra JF. Complications of laparoscopic gynecologic surgery. JSLS. 2014; 18: e2014.00058.

3. Abilez O, Picquet J, Zarins C, Tarin T, Hsu T, Shelton A. Complications in laparoscopy. In: Nezhat C, Nezhat F, Nezhat C (authors). Nezhat's Operative gynecologic laparoscopy and hysteroscopy. Cambridge: Cambridge University Press; 2008. pp. 520-536.

4. Stovall TG, Mann WJ. Complications of gynecologic laparoscopic surgery. Up To Date. 2009; 17.

5. Alkatout I. Complications of laparoscopy in connection with entry techniques. J Gynecol Surg. 2017; 33: 81-91.

6. Krishnakumar S, Tambe P. Entry complications in laparoscopic surgery. J Gynecol Endosc Surg. 2009; 1: 4-11.

7. van der Voort M, Heijnsdijk EA, Gouma DJ. Bowel injury as a complication of laparoscopy. Br J Surg. 2004; 91: 1253-1258.

8. Llarena NC, Shah AB, Milad MP. Bowel injury in gynecologic laparoscopy: a systematic review. Obstet Gynecol. 2015; 125: 1407-1417.

9. Montz FJ, Holschneider CH, Munro MG. Incisional hernia following laparoscopy: a survey of the American Association of Gynecologic Laparoscopists. Obstet Gynecol. 1994; 84: 881-884.

10. Fritton, K, Tanner EJ. Complications of minimally invasive surgery. In: Ramirez P, Frumovitz M, Abu-Rustum N. Principles of gynecologic oncology surgery. Philadelphia, PA: Elsevier; 2018. pp. 380-390.

11. Kiran A, Hilton P, Cromwell DA. The risk of ureteric injury associated with hysterectomy: a 10-year retrospective cohort study. BJOG. 2016; 123: 1184-1191.

12. Nieboer TE, Johnson N, Lethaby A, Tavender E, Curr E, Garry R et al. Surgical approach to hysterectomy for benign gynaecological disease. Cochrane Database Syst Rev. 2009; 3: CD003677. 